\title{
A Bipolar Traffic Density Awareness Routing Protocol for Vehicular Ad Hoc Networks
}

\author{
David Chunhu Li, ${ }^{1}$ Li-Der Chou, ${ }^{1}$ Li-Ming Tseng, ${ }^{1}$ Yi-Ming Chen, ${ }^{2}$ and Kai-Wei Kuo ${ }^{1}$ \\ ${ }^{1}$ Department of Computer Science and Information Engineering, National Central University, No. 300 Jhongda Road, \\ Jhongli City, Taoyuan County 32001, Taiwan \\ ${ }^{2}$ Department of Information Management, National Central University, No. 300 Jhongda Road, Jhongli City, \\ Taoyuan County 32001, Taiwan
}

Correspondence should be addressed to Li-Der Chou; cld@csie.ncu.edu.tw

Received 29 August 2014; Accepted 1 September 2014

Academic Editor: David Taniar

Copyright (C) 2015 David Chunhu Li et al. This is an open access article distributed under the Creative Commons Attribution License, which permits unrestricted use, distribution, and reproduction in any medium, provided the original work is properly cited.

\begin{abstract}
To support an increasing amount of various new applications in vehicular ad hoc networks (VANETs), routing protocol design has become an important research challenge. In this paper, we propose a Bipolar Traffic Density Awareness Routing (BTDAR) protocol for vehicular ad hoc networks. The BTDAR aims at providing reliable and efficient packets delivery for dense and sparse vehicle traffic network environments. Two distinct routing protocols are designed to find an optimal packet delivery path in varied vehicular networks. In dense networks, a link-stability based routing protocol is designed to take vehicles connectivity into consideration in its path selection policy and maximize the stability of intervehicle communications. In sparse networks, a min-delay based routing protocol is proposed to select an optimal route by analyzing intermittent vehicle connectivity and minimize packets delivery latency. Intervehicles connectivity model is analyzed. The performance of BTDAR is examined by comparisons with three distinct VANET routing protocols. Simulation results show that the BTDAR outperforms compared counterpart routing protocols in terms of packet delivery delay and packet delivery ratio.
\end{abstract}

\section{Introduction}

Recent developments in the field of machine-to-machine communication (M2M) have led to a renewed interest in vehicular communications. The importance of communication protocols in vehicular ad hoc networks (VANETs) has long been recognized. In the automotive industry, approximately $60 \%$ of new cars are expected to be equipped with connected car solutions by 2017, according to the report in [1]. Vehicle-to-vehicle (V2V) and vehicle-to-infrastructure (V2I) communication in vehicular networking endows customers with new exciting services and driving experiences. To achieve reliable vehicular communications, various sets of short-range wireless protocols and standards have been developed. Dedicated short-range communication (DSRC) systems are widely used in Europe, Japan, and the United States [2]. Electronic toll collection is one popular application of DSRC systems. Other possible applications of intelligent transportation system (ITS) include intersection collision avoidance, probe data collection, and cooperative adaptive cruise control $[3,4]$. Telematics, which is a term that refers to the integrated use of telecommunication and informatics, generally involves two fields [5]. The first field is the convergence of information processing and telecommunications and includes applications and automation in vehicles, such as GPS (global positioning system) navigation control and automatic driving assistance systems. The second domain refers to any universal applicable wireless technologies and computation systems that can enhance ITS. The IEEE standard in the 802.11 families (e.g., IEEE802.11b and IEEE801.11p) [6] to address wireless access in vehicular environments (WAVE) is a primary example of the second field.

One of the most significant current discussions in vehicular networking is designing a reliable communication protocol in both data link layer and network layer. Routing protocols are the central focus of the study and developing 


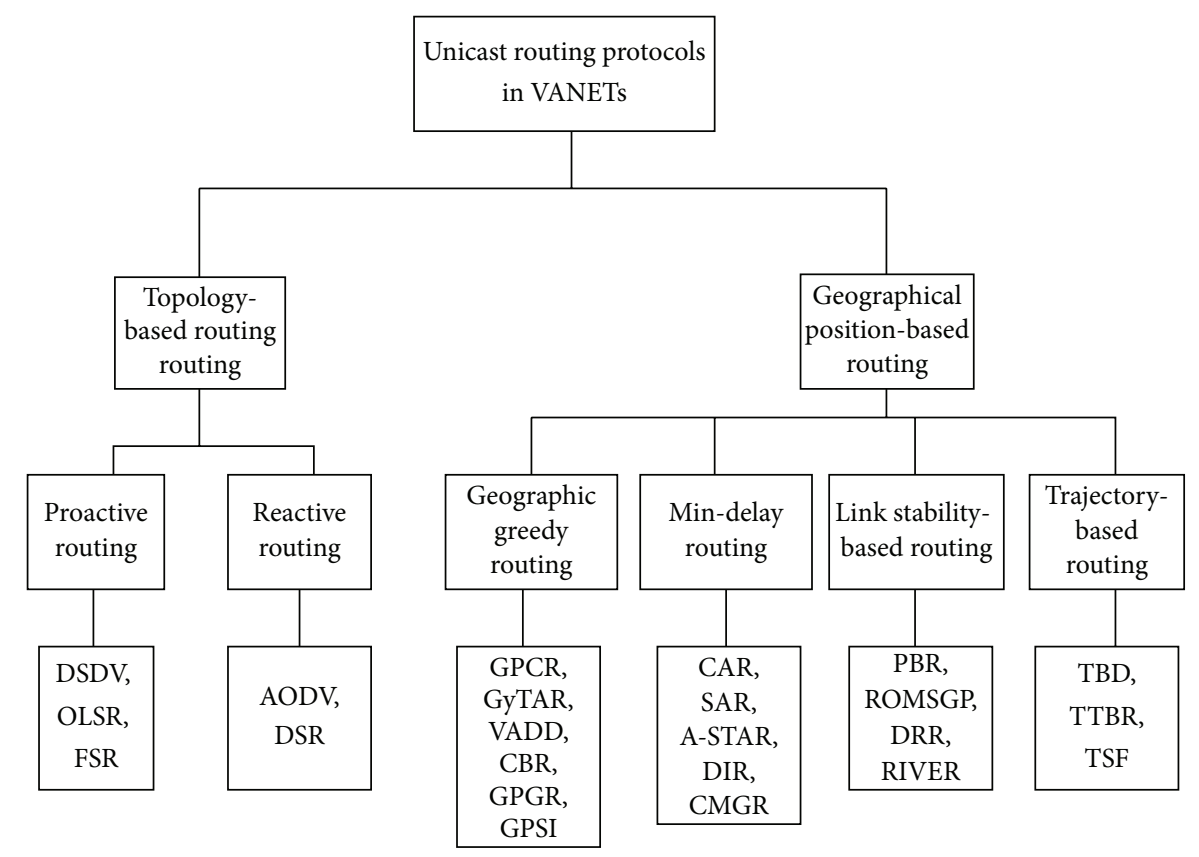

FIGURE 1: Taxonomy of unicast routing protocols in vehicular ad hoc networks.

an intelligent and dynamic routing protocol is necessary to cope with complex and highly mobile environments in VANETs. A variety of studying strategies have been proposed for designing a routing protocol. Routing protocols in VANETs can generally be divided into three categories: broadcast, multicast, and unicast protocol, each of which has its advantages and shortcomings. Flooding, a common strategy broadly used in broadcast routing protocol, disseminates, and broadcasts messages to all vehicles in vehicular networks. Although extensive ranges of applications are achieved with broadcast routing protocol, redundant message delivery and bandwidth waste are unavoidable. Moreover, broadcast storm is another major challenge. Multicast routing can improve the efficiency of bandwidth usage by employing most reliable packet delivery via multipaths. Message delivery path selections in multicast routing are generally categorized into two classes: application dependence and application independence. Communication bandwidth utilization in unicast routing protocol is more efficient than the other two categories, whereas the vehicular network infrastructure requirement is very strict in unicast routing protocol. An efficient unicast routing protocol is capable of reliably delivering a data packet between source and destination vehicles with satisfying basic application quality requirements, such as delay and packet delivery ratio. Much research has assumed that sufficient intermittent relay vehicles and network infrastructures assist in delivering data packets. Moreover, many routing algorithms and protocols in the literatures are designed to provide packets delivery with assumption of static traffic density. Researchers [7-9] have raised concerns that such rigorous assumptions may prevent practically applying routing protocols in real life. In particular, traffic density dramatically varies in different places all the time. Even in a certain location the traffic density differs during rush hour and off-peak time. Thus, a unicast routing protocol should be able to flexibly and intelligently adapt to a complex vehicular network environment. For this reason, this work proposes intelligent bipolar traffic density awareness routing protocols for VANETs. The proposed routing protocol solves the problems regarding effectively delivering data packets in diverse vehicular networks whereas traditional VANET routing protocols make this difficult.

The rest of the paper is organized as follows. Section 2 reviews related literatures on unicast routing protocol in VANETs. Section 3 analyze intervehicles connectivity model with three important factor affecting packet delivery path qualities. The proposed bipolar traffic density awareness routing protocol is presented in Section 4. Section 5 discusses simulation and result analysis and is finally followed by a conclusion to the work.

\section{State of the Art}

Data packet delivery in wireless vehicular network communication has suffered from unstable delivery paths, rapid changes in relay nodes, and intermittent connections among relay vehicles. Therefore, many researchers have conducted studies on designing various routing protocols for diverse applications [10-14]. Unicast routing protocol has been an important research issue. Figure 1 illustrates the taxonomy of unicast routing protocols in VANETs. We can broadly classify unicast routing protocols for VANETs into two categories: topology-based routing and geographical locationbased routing. Recent literatures $[15,16]$ indicate that the geographical position-based routing protocol is more attractive than the topology-based routing protocol because of its good 
performance and merits in terms of packet delivery delay and packet delivery ratio.

The geographical position-based routing can be further classified into four subcategories based on strategies of selecting packets delivery path. There are some previous works that use greedy strategies and forward the packets closer to the destination by using local information in VANETs. For instance, Wu et al. [17] combine greedy algorithm and predictive approach to design a geographic routing (GPSI) for VANET. The GPSI consists of two modes forwarding packets: greedy mode and predictive mode. In greedy mode, a weight value combining the distance and multirate is used to select relay nodes. In predictive mode, the GPSI predicts the direction of vehicles to choose next relay nodes. In mindelay routing, routing protocols are designed to minimize average packet delivery latency and forward the packets as soon as possible in various environments [18]. How to find a reliable packet delivery route between the source and destination nodes to improve packet delivery ratio is the principal objects in link stability-based routing $[19,20]$. Different from aforementioned three categories of routing protocols, the packets are forwarded along a predefined curve in network topology with the trajectory-based routing [21].

Except for classification of routing protocols mentioned above, there are some routing protocols proposed for some particular environments (e.g., intersections and road junctions) in vehicular networks. Roadside units (e.g., access points and traffic lights) are broadly utilized to assist packet forwarding in complex environments [22]. Intersectionbased routing protocols are proposed to provide efficient packets delivery in urban city environments, such as the works in [23-25] and IBR in [26]. In IBR, the packets are forwarding among intersections with a carry-and-forward manner in a sparse traffic vehicular network. Junction-based routing protocols, for instance, JARR in [27] and JTAR [28], are designed to address the deficiencies in packet forwarding of current routing protocols. In JARR, the packets are routed from one junction to another according to the position and direction of next available hops. Traffic density in selected next junction is estimated to avoid the problem of network topology hole.

Unlike previous works in the literatures, this work analyzes intervehicles connectivity models with three important factors that affect packet delivery paths. Notably, vehicles relative velocity, intervehicles link lifetime, and average packets waiting time in storage buffer queue are derived to assist the vehicles to choose appropriate relay nodes. Accordingly, two variants of routing protocols are designed to support applications for both dense and sparse traffic density scenarios, respectively. Instead of simply evaluating performance of routing protocols with ordinary metrics, such as packet delivery delay and packet delivery ratio, an extensive simulation with a real map is conducted in this work to access packet delivery efficiency in terms of control packets and channel access. The simulation results showed that the proposed routing protocol outperforms compared three representative routing protocols in the literatures.

\section{Intervehicles Connectivity Model}

This section shows the development of intervehicle connectivity model in vehicular communication. We analyze the dynamics of intervehicle communication links in vehicular multihop networks.

3.1. Assumption. To begin, the following is assumed to be reasonable. Each vehicle in a wireless vehicular network is implemented with an on-board-unit (OBU) that integrates the IEEE802.11 family DSRC wireless communication system. The vehicles are equipped with GPS devices that can provide information about real-time vehicle speed, direction, and land attributes. The vehicles can also proactively and periodically collect real-time traffic density information and compare the average intervehicle space with minimum safe following distance to gain information regarding overall traffic congestion. The average intervehicle space is described in [29] and expressed by

$$
S=\alpha+\beta V+\gamma V^{2},
$$

where $\alpha$ is effective vehicle length, $\beta$ is drivers' interaction time, $\gamma$ is the reciprocal of vehicle maximum deceleration, and $V$ is real-time vehicle velocity. The value of $\alpha$ is known and the maximum value of $\beta$ is 1.8 seconds according to the study in [30]. For simplicity, all vehicles are assumed to have the same maximum deceleration. With consideration of various vehicle mobility patterns, (1) can be modified as

$$
S=\alpha+\left(\beta+\bar{X}_{t}\right) V .
$$

$\bar{X}_{t}$ is the interarrival time of vehicles that pass a certain monitor on the road. The distribution of vehicle interarrival time in vehicular networks is assumed to be Poisson distribution [31]. The value of $\bar{X}_{t}$ is then calculated as follows and $\lambda_{1}$ is the interarrival rate of vehicles that pass a certain monitor on the road that is available by collecting the statistical data, which is estimated by

$$
E\left(\bar{X}_{t}\right)=\operatorname{Var}\left(\bar{X}_{t}\right)=\lambda_{1} .
$$

Therefore, with the knowledge of interarrival time $\bar{X}_{t}$, the average intervehicle space of vehicle networks is predictable. When the source vehicle compares the average intervehicle space with the minimum following distance, the overall traffic density in vehicle networks is acquired. Accordingly, the source vehicles may dynamically adjust suitable routing algorithms for various traffic conditions to achieve efficient packet delivery.

3.2. Vehicle Connectivity Model. This section discusses the connectivity model of intervehicle communication for analyzing the dynamics of communication links. Analytical expressions are derived to characterize intervehicular communications with the three most important principal factors that affect packet delivery path quality [32] including relative intervehicle velocity, link lifetime of intervehicle communication, and average waiting time of packets in 


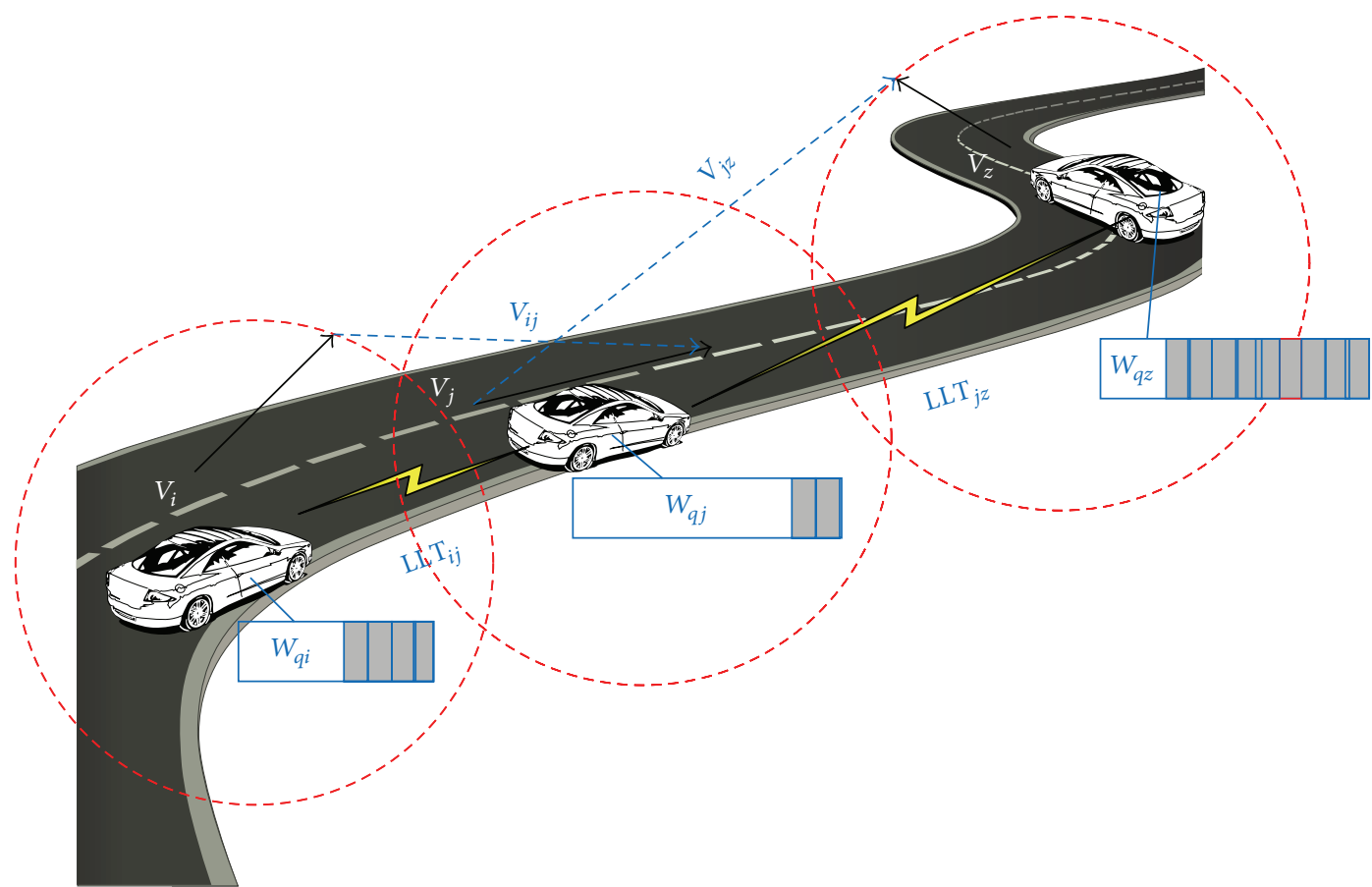

FIGURE 2: Intervehicle connectivity model (where $V_{x y}$ is vehicles relative velocity, LLT $_{x y}$ is intervehicle communication link lifetime, and $W_{q x}$ is packets waiting time in storage buffer, $x, y=i, j, z)$.

vehicle storage buffer queue. Figure 2 depicts the intervehicle communication connectivity model. Each vehicle has various communication abilities that affect the connectivity between the neighbouring nodes.

In VANETs, any two neighbouring vehicles have different real-time driving velocities and directions at all times. The communication quality is highly affected by the rapid changes of the relative positions of the two vehicles. Given the coordinates of two vehicles $i$ and $j$ in a two-dimensional space as $\left(x_{i}, y_{i}\right)$ and $\left(x_{j}, y_{j}\right)$, if vehicle velocities of two vehicles $V_{i}$ and $V_{j}$ have a large difference or two vehicles are moving to opposite driving directions, the communication between these two vehicles is soon disconnected. Thus, the relative intervehicle velocity $V_{r}$ can be obtained by

$$
V_{r}=\frac{d \theta}{d t} \times \frac{S_{d}^{2}}{\alpha}
$$

$d \theta / d t$ is the rate of change relative angle between two vehicles. $S_{d}$ is the line of sight distance of two vehicles and a function of time $t$ that can be evaluated by

$$
\begin{aligned}
& S_{d}(t) \\
& =\left(\left(\left(x_{j}-x_{i}\right)+t\left(V_{j} \cos \theta_{j}-V_{i} \cos \theta_{i}\right)\right)^{2}\right. \\
& \left.+\left(\left(y_{j}-y_{i}\right)+t\left(V_{j} \sin \theta_{j}-V_{i} \sin \theta_{i}\right)\right)^{2}\right)^{1 / 2} \text {. }
\end{aligned}
$$

The $S_{d}(t)$ has a minimum value when

$$
t=\left(\left(V_{j} \cos \theta_{j}-V_{i} \cos \theta_{i}\right)\left(x_{i}-x_{j}\right)\right.
$$

$$
\begin{gathered}
\left.+\left(V_{j} \sin \theta_{j}-V_{i} \sin \theta_{i}\right)\left(y_{i}-y_{j}\right)\right) \\
\cdot\left(\left(V_{j} \cos \theta_{j}-V_{i} \cos \theta_{i}\right)^{2}+\left(V_{j} \sin \theta_{j}-V_{i} \sin \theta_{i}\right)^{2}\right)^{-1} .
\end{gathered}
$$

Accordingly, the minimum threshold value of relative intervehicle velocity $V_{r t h}$ is obtained. There is a positive relationship between the link lifetime and communication path quality. The link lifetime of any two neighbouring vehicles is related to vehicle velocity, direction, and position. In a two-dimensional plane, consider two vehicles $i$ and $j$ that has a transmission range of $R$, vehicle speeds $V_{i}$ and $V_{j}$, and coordinates $\left(x_{i}, y_{i}\right)$ and $\left(x_{j}, y_{j}\right)$, respectively. The link lifetime [33] of two vehicles communication is predicted by

$$
\operatorname{LLT}(t)=\frac{-(a b+c d)+\sqrt{\left(a^{2}+c^{2}\right) R^{2}-(a d-b c)^{2}}}{a^{2}+c^{2}},
$$

where $a=\left(V_{i} \cos \theta_{i}-V_{j} \cos \theta_{j}\right) t, b=x_{i}-x_{j}, c=\left(V_{i} \sin \theta_{i}-\right.$ $\left.V_{j} \sin \theta_{j}\right) t$ and $\mathrm{LLT}_{\max }$.

Further analysis of (7) shows that with the known real time of two vehicles' mobility information, the link lifetime LLT is a function of time $t$. Thus, with a specific time $t$, the expected communication link lifetime between two vehicles is calculated. As the maximum threshold of LLT only exists when the time is indefinite, the data packet time-to-live (TTL) is chosen as the maximum threshold of LLT, LLT max $_{\text {. }}$

Every vehicle is confronted with a different vehicular network communication environment. Some vehicles may play the role of relay nodes while others do not. In addition, vehicles have finite storage buffers to temporarily store 
received data packets. Thus, the average data packet waiting time in the buffer queue of vehicles varies. To avoid severe contention of selecting relay vehicles that have congested data traffic, the average data packet waiting time in vehicles is analyzed. Again, the distribution of interarrival time of the data packet in the buffer queue of vehicles is assumed to be Poisson distribution. The process service of the buffer queue is then represented with a Single-Server Queue with Poisson Input and General Service (M/G/1) Markov process queue. The cumulative distribution function of the average waiting time of a packet in the buffer queue is expressed by

$$
W_{q}(t)=1-\rho e^{-\mu(1-\rho) t} .
$$

The probability $P_{q}$ that the waiting time $W_{q}$ of a packet exceeds the maximum predefined waiting time $W_{\max }$ of a specific queue is expressed by

$$
P_{q}=P\left(W_{q}>W_{\max }\right)=\frac{r^{c} e^{-\left(c \mu-\lambda_{2}\right)}}{c !(1-\rho)},
$$

where $\lambda_{2}$ is the arrival rate of the packet, $\mu$ is the service rate of processing the packet, $c$ is the number of service channels that process the packet, and $r=\lambda_{2} / \mu, \rho=r / \mu$. Using the analysis connectivity model of intervehicle communication, two variants of routing protocols are proposed to efficiently and reliably deliver data packets in both nonsparse and sparse traffic density environments, respectively. Two distinct routing algorithms are designed to discover the optimal packet delivery path in varied vehicular network environments.

\section{Bipolar Traffic Density Awareness Routing Protocol}

Since the intervehicle connectivity including relative speed, the link lifetime of communication, and the probability that packets waiting time exceeds the predefined threshold are known, two types of routing algorithms are designed for dense traffic density and sparse traffic density vehicular networks, respectively. See all symbols with their definitions in the Abbreviation section.

\subsection{BTDAR-R: Routing Algorithm for Dense Traffic Density} Vehicular Networks. This section proposes the routing algorithm BTDAR-R for dense traffic density vehicular networks. BTDAR-R is a type of link-stability based routing protocol in VANETs. In dense traffic density vehicular networks, the packet delivery path is discovered according to the quality of the path. Packets are transferred among the vehicles via a multihop relay manner. Many candidate relay nodes lie between the source and destination vehicles and the source vehicle must find the optimal packets delivery path to reduce packet delivery end-to-end delay while satisfying stability and other quality of service requirements. The optimal packet delivery path consists of a series of relay vehicles that have minimum average relative intervehicle velocity, maximum average link lifetime, and minimum probability that average packet waiting time exceeds the predefined maximum waiting time in the storage queue of vehicles. To represent

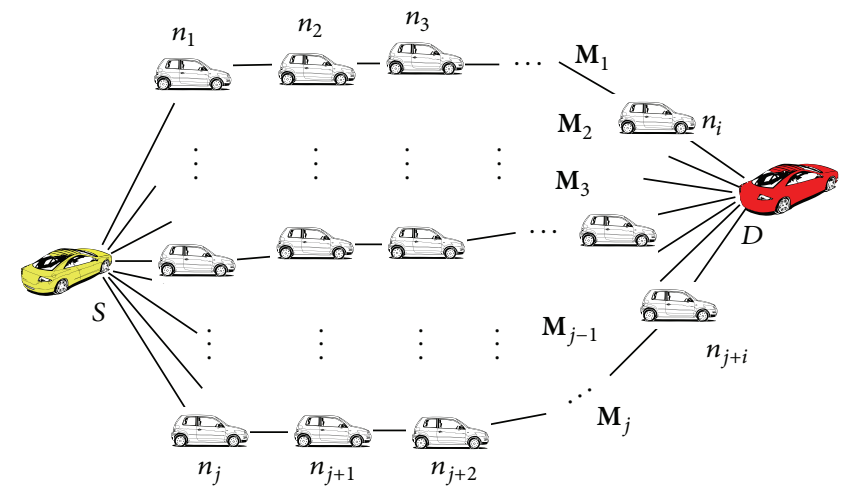

FIGURE 3: Selection of an optimal packet delivery path.

the quality of the selective packet delivery path, this study defines a packet delivery path quality matrix $\mathbf{M}$ to contain the information related to the three principal factors discussed in Section 3. Figure 3 illustrates the selection of an optimal packet delivery path among relay nodes in the vehicular networks. Suppose that a source vehicle $S$ is going to discover the packet delivery path to the destination vehicle $D$. Each candidate path between $S$ and $D$ contains a path quality matrix $\mathbf{M}_{j}(j=1,2,3, \ldots, n)$ as shown in the following:

$$
\mathbf{M}_{j}=\left[\begin{array}{ccccc}
V_{r 1} & V_{r 2} & V_{r 3} & \cdots & V_{r i} \\
\mathrm{LLT}_{1} & \mathrm{LLT}_{2} & \mathrm{LLT}_{3} & \cdots & \mathrm{LLT}_{i} \\
P_{q 1} & P_{q 2} & P_{q 3} & \cdots & P_{q i}
\end{array}\right] .
$$

The path quality matrix $\mathbf{M}$ is a 3-by- $n$ matrix in which the entries in each row represent the path quality effecting factors discussed in the previous section. A path quality factor threshold matrix $\mathbf{A}$ is defined in (11) and an $n$-by- 1 matrix $\mathbf{N}$ with one column is used for the selection of optimal path. The entry of each row in matrix $\mathbf{N}$ is the reciprocal of value $n_{r}$, which is the total number of relay nodes on a candidate path between the source and destination vehicles. Consider

$$
\mathbf{A}^{\mathbf{T}}=\left[\begin{array}{lll}
V_{r_{-} t h} & \operatorname{LLT}_{\max } & P_{q_{-} \min }
\end{array}\right] .
$$

Accordingly, the path quality matrix $\mathbf{M}$ is multiplied by $\mathbf{N}$ for each candidate selective path. The multiplication matrices $\mathbf{M}$ and $\mathbf{N}$ are further compared with a threshold path quality matrix A. Thus, an optimal packet delivery path is calculated with the selection of minimum relative vehicle velocity, maximum communication link lifetime, and minimum probability that average packet waiting time exceeds the predefined maximum waiting time in the storage buffer queue of vehicles.

\subsection{BTDAR-P: Routing Algorithm for Sparse Traffic Density} Vehicular Networks. In sparse traffic density vehicular networks, packet delivery is highly affected by the intermittently connected sparse vehicles. Thus, a relay vehicle must temporarily store the received packets and forward them once the relay vehicle is in the communication range of other neighbouring vehicles. Although the carry-to-forward strategy is popularly adopted in many VANET routing protocols 


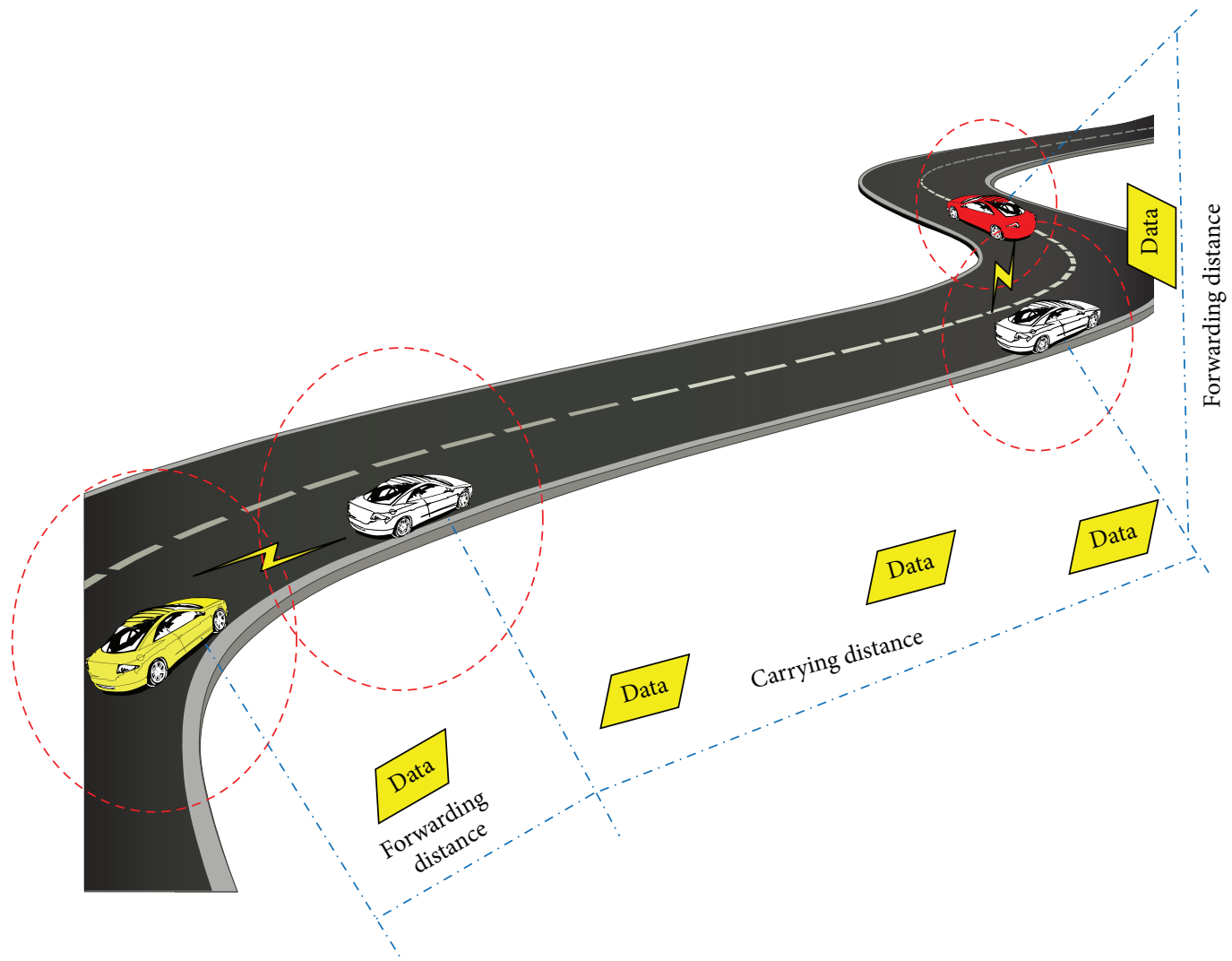

FIGURE 4: Connectivity model in sparse traffic density vehicular networks.

in the literatures $[34,35]$, improving the stability and efficiency of routing protocols in complex sparse traffic density vehicular networks remains a challenge. Thus, this study has designed a min-delay based routing algorithm BTDAR$\mathrm{P}$ for sparse traffic density vehicular networks. Figure 4 shows the analysis of the intermittent connectivity among vehicles in sparse traffic density. The connectivity consists of packet forwarding distance and packet carrying distance. Accordingly, packet forwarding delay and carry delay occur in each type of distance. In a sparse traffic vehicular network, a source vehicle must periodically broadcast hello beacons to learn the position of any possibly connected neighbouring vehicles. Once a temporary relay vehicle is connected, packet delivery should be accomplished as soon as possible to prevent unexpected disconnection due to sparse traffic density. The illustration of a simple example in Figure 4, where there is only one relay vehicle between the source and destination vehicle, can assist the reader in understanding the calculation of forwarding distance and carrying distance and then be extended to the entire sparse vehicular network.

Suppose the road length between three connected vehicles is $l$. It is easily understood that $l$ is the sum of packet forwarding distance $l_{f}$ and carrying distance $l_{c}$. The forwarding distance is expressed by

$$
l_{f}=\sum_{i=1}^{n} \int_{x}^{R} S_{d}(t) \cdot P_{c} \cdot x d x
$$

$P_{c}$ is the contacted probability that there is a relay vehicle connected with the source vehicle. $S_{d}(t)$ is the line of sight distance of two contacted vehicles, which is calculated with (5). $n$ is the total number of packet forwarding times, and $x$ is the current position of the relay vehicle at a certain time $t$. All vehicles are assumed to be equipped with the same wireless communication network card for which the maximum range is $R$ meters. In sparse vehicle networks, to achieve stable data packet delivery, both source vehicle and relay vehicle should move to the same region of interest. Although some literature states that choosing relay vehicles in opposite lanes may reduce packet broadcast delay, applying the same principle is impractical for unicasting packets in a sparse traffic vehicular network. When two vehicles enter the communication range, the packets forwarding region is dynamic among the region of interest. Assuming that the area of packet forwarding region $l_{c}$ and the area of region of interest $A_{i}$ are known during two vehicles' communication, the probability of there being two contacted vehicles at a certain time $t$ is obtained by

$$
P_{c}=\frac{1}{A_{i}} \int_{t_{1}}^{t_{2}}\left(\sum_{j=1}^{n} A_{f j}(t)-\sum_{j=1}^{n-1} \sum_{k=j+1}^{n} A_{f j k}(t)\right) d t .
$$

$t_{1}$ is the time that the vehicle enters the area of region of interest and $t_{2}$ is the time that the vehicle leaves the area of region of interest. Thus, the packet carrying distance is calculated with $l-l_{f}$. The carrying delay $d_{c}$ of the packet 


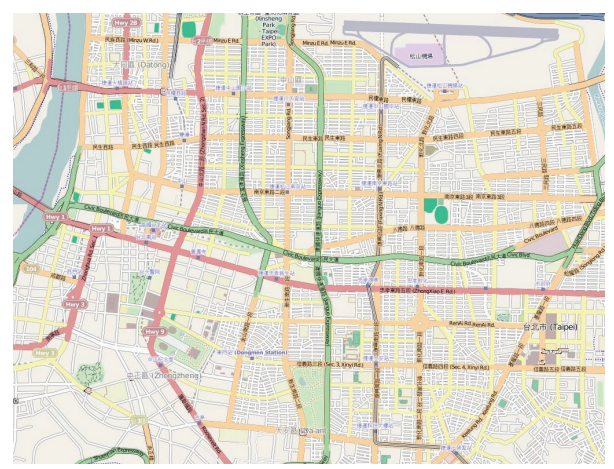

(a) Urban area selected from OpenStreetMap

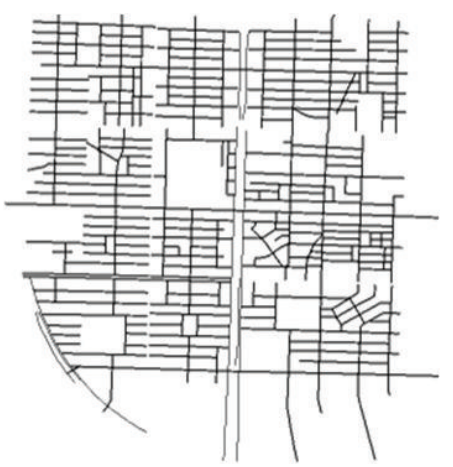

(b) Generated topology network for selected urban area (SUMO view)

FIGURE 5: Real terrain maps in simulations.

delivered between the relay vehicle and destination vehicle is calculated by $d_{c}=l_{c} / \bar{v}$. The forwarding delay consists of four basic types of delays. The dominant delay component in forwarding delay is the queuing delay $d_{q}$, which can be represented by (8). With forwarding delay and carrying delay, the total end-to-end packet delay in a selective path is obtained. Therefore, an optimal packet delivery path in a sparse traffic vehicular network is chosen with the minimum average end-to-end packet delivery delay.

\section{Simulation and Discussion}

The performance of the proposed bipolar traffic density awareness routing protocol BTDAR is examined by a series of simulations. These simulations cover the two proposed variants of traffic density awareness routing protocols BTDAR$\mathrm{R}$ and BTDAR-P using NS2 [36] and compare them with three representative routing protocols for vehicular networks in the literature, GPSI [17], IBR [26], and JARR [27]. Details of the simulation environment are listed in Table 1. SUMO described in [37], which is a famous and acknowledged mobility generator in VANETs simulation, is used to generate realistic vehicle mobility patterns. A real area is selected from the freely available digital map, OpenStreetMap [38], and SUMO automatically exports a simulation topology, as shown in Figure 5. The select area is from the downtown area of Taipei with various numbers for vehicle, ranging from 50 to 500 . To discuss the effect of vehicle density, with the same of road area, the vehicle density is changed due to the different number of vehicles. The average vehicle density is varied from 2 vehicles $/ \mathrm{km}$ to 20 vehicles $/ \mathrm{km}$. The effect of vehicle velocity is also explored. The velocity of each vehicle is changed $3 \mathrm{~m} / \mathrm{s}$ to $30 \mathrm{~m} / \mathrm{s}$. The simulation duration of each round is 2000 seconds. Each simulated result is obtained from mean value of 100 rounds to achieve a high confidence level in the results. The confidence level was set to $95 \%$. The mobility model of vehicles within a $4000 \mathrm{~m} \times 3000 \mathrm{~m}$ area is the Intelligent Driving Model with Lane Change (IDMLC). Extensive simulations evaluated the various impacts
TABLE 1: Simulation environments.

\begin{tabular}{|c|c|}
\hline Simulation parameter & Value \\
\hline Vehicle density & $\begin{array}{l}2 \text { vehicles } / \mathrm{km}- \\
20 \text { vehicles } / \mathrm{km}\end{array}$ \\
\hline Vehicle velocity & $3 \mathrm{~m} / \mathrm{s}-30 \mathrm{~m} / \mathrm{s}$ \\
\hline Number of lanes & 3 lanes \\
\hline MAC protocol & IEEE802.11p \\
\hline $\begin{array}{l}\text { Wireless communication } \\
\text { range }\end{array}$ & 250 meters \\
\hline Beacon interval & 1.0 second \\
\hline Data traffic & $\begin{array}{c}\text { Constant bit rate } \\
(\mathrm{CBR})\end{array}$ \\
\hline Data packet size & 512 bytes \\
\hline $\begin{array}{l}\text { Maximum comfortable } \\
\text { acceleration }\end{array}$ & $2 \mathrm{~m} / \mathrm{s}^{2}$ \\
\hline $\begin{array}{l}\text { Maximum comfortable } \\
\text { deceleration }\end{array}$ & $2 \mathrm{~m} / \mathrm{s}^{2}$ \\
\hline Mobility model & IDM-LC \\
\hline Simulation time & 2000 seconds \\
\hline
\end{tabular}

of vehicle traffic density and vehicle velocity. The observed metrics of interest are:

Packet delivery delay is the average time that data packets are sent from the sources to the destinations.

Packet delivery ratio is the number of data packets successfully received by the destinations divided by the total number of data packets sent from the sources.

Number of control bytes transmitted per data byte delivered is the ratio of control bytes and data bytes that is an indicator to evaluate how the routing protocols efficiently utilize control packets in packet delivery. 


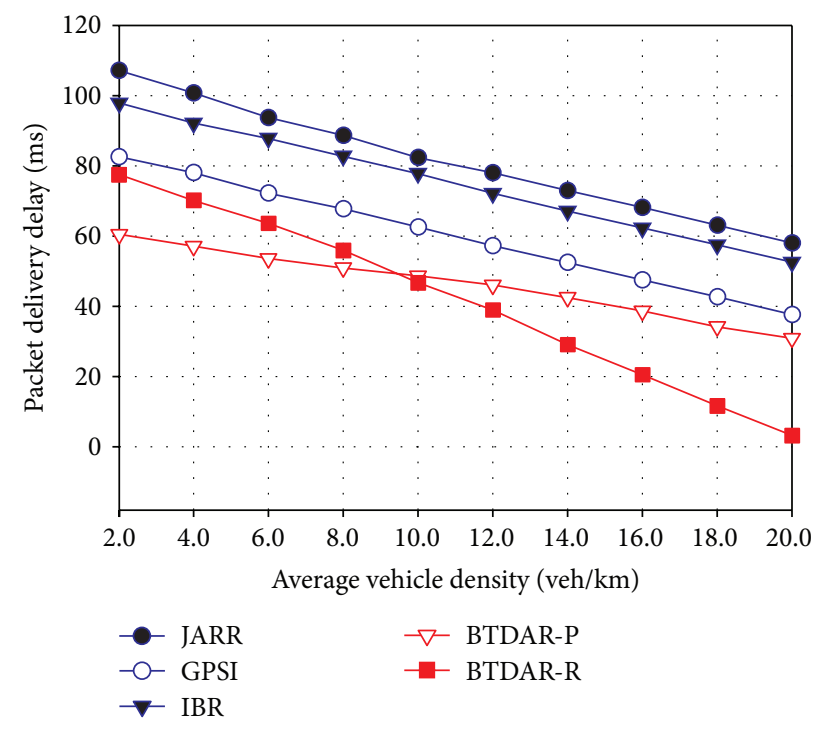

FIGURE 6: Packet delivery delay as a function of average vehicle density.

Number of total packets transmitted per data packet delivered is the number of all packets (i.e., control packets and data packets) transmitted divided by the total number of data packets delivered to the destinations. This measure shows the efficiency of routing protocols in terms of channel access in a contention-based wireless ad hoc network.

5.1. Packet Delivery Delay. As shown in Figure 6, all simulated routing protocols demonstrate the property that the packet delivery delay decreases as the number of vehicles increases. The reason for this phenomenon is that the packet delivery path is easily selected when the vehicle density increases. It is observed that the BTDAR-R and BTDAR$\mathrm{P}$ achieve the lowest packet end-to-end delay in dense and sparse networks, respectively. This achievement is due to the fast and efficient path selection strategy proposed in BTDAR along the lane. In particular, in the dense network, the BTDAR-R can quickly pick up an optimal packet delivery path between the source and destination vehicle. Since the connectivity model in BTDAR considers the impact of mobility and communication ability of vehicles, such as relative speed, lifetime of link, and congestions in the data storage buffer, the time spent on path discovery is significantly reduced because those fast moving vehicles and hot spot vehicles are ignored as relay nodes. Thus, BTDAR$\mathrm{R}$ can avoid long delays caused by significant amounts of calculation time spent on overwhelmed vehicles. On the other hand, in the sparse network, the BTDAR-P can quickly decide a packet delivery path between the source and destination vehicle by minimizing the various delay components. Particularly, various types of delay that occur during the forwarding and carrying on to relay vehicles are analyzed to discover an optimal packet delivery path. The average hop number that the packets forwarded in various vehicle densities is reduced in the BTDAR-P and BTDAR-R, which contributes to decrease of average packet delivery delay. The

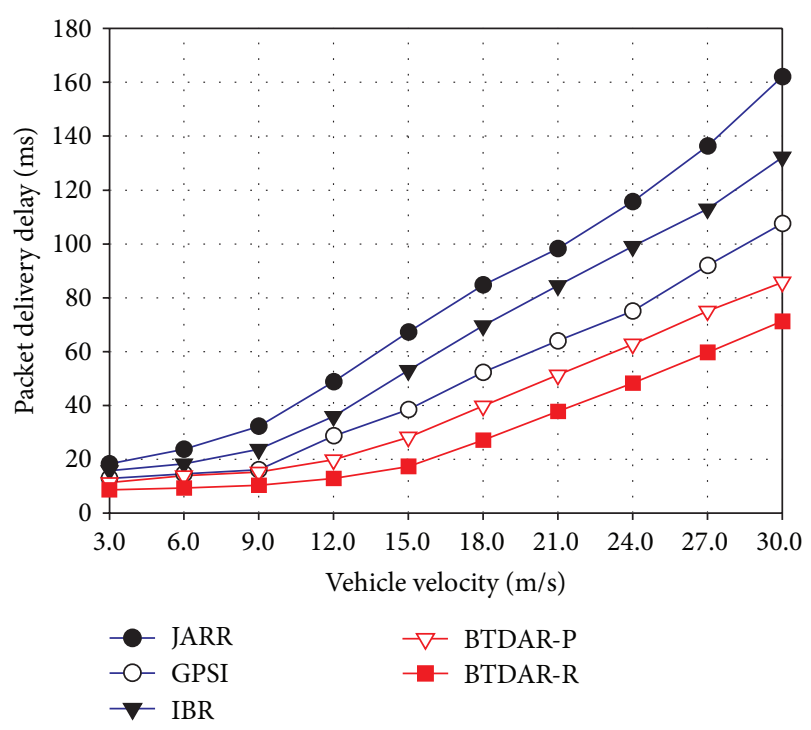

FIGURE 7: Packet delivery delay as a function of vehicle velocity.

other three counterpart routing protocols experience long packet delivery delays in both sparse and dense networks when compared with these two variants of BTDAR. In the sparse vehicular networks, when the vehicle density is 2 vehicles $/ \mathrm{km}$, the BTDAR-P can decrease the packet delivery delay up to $43.6 \%$ compared to JARR, $26.8 \%$ and $38.2 \%$ against GPSI and IBR, respectively. In the dense vehicular networks, when the vehicle density is 20 vehicles $/ \mathrm{km}$, the BTDAR-R can significantly reduce the packet delivery delay up to $94.4 \%$ compared to JARR, $89.5 \%$ and $93.8 \%$ against GPSI and IBR, respectively.

Figure 7 shows the comparison of performance versus vehicle velocity. All simulated routing protocols show that packet delivery delay continues to increase as the vehicle speed becomes faster. In both sparse and dense vehicular networks, BTDAR-P and BTDAR-R outperform all three other routing protocols. This result occurs because relay vehicles are selected with consideration of mobility patterns. Relative velocities among forwarding vehicles are especially calculated and compared to avoid unstable packet delivery paths. Thus, the BTDAR can significantly reduce the delays caused by path discovery. When the vehicle velocity is lower than $12 \mathrm{~m} / \mathrm{s}$, packet delivery delay experiences no significant increase for all five routing protocols. However, once the vehicle velocity is faster than $12 \mathrm{~m} / \mathrm{s}$, the packet delivery delay grows rapidly; the faster the vehicle speed, the more packet delivery delay increases. The findings of this simulation confirm that vehicle velocity imposes a negative impact on packet delivery delay. When compared with JARR, GPSI, and IBR, the proposed BTDAR protocols demonstrate better performance on packet delivery delay with the vehicle velocity as a function.

5.2. Packet Delivery Ratio. From Figure 8, BTDAR-R and BTDAR-P achieve the highest packet delivery ratio when compared with the three other routing protocols in both dense and sparse traffic density vehicular networks. In the sparse vehicular networks, when the vehicle density is 


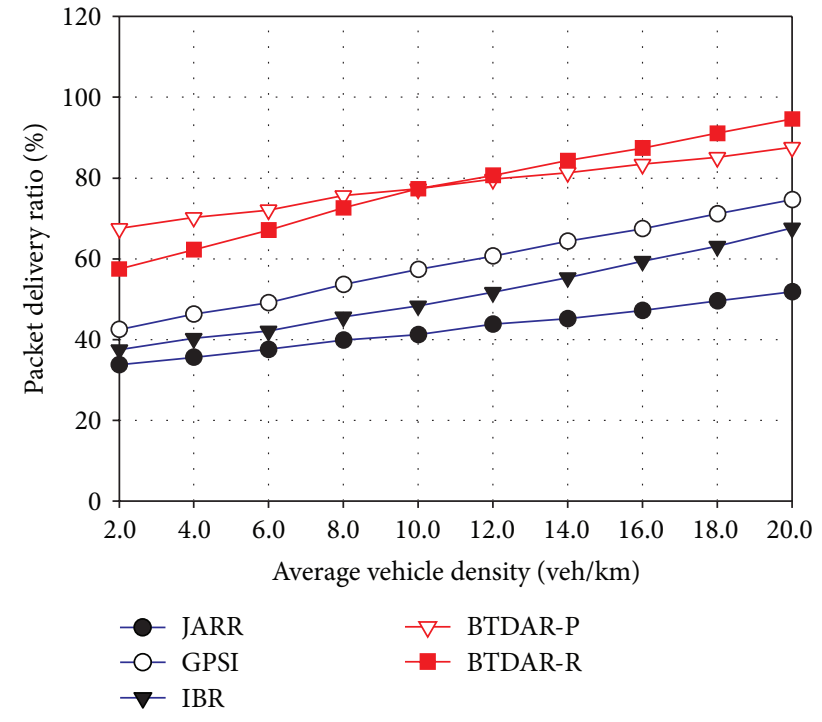

Figure 8: Packet delivery ratio as a function of average vehicle density.

2 vehicles/ $\mathrm{km}$, the BTDAR-P can improve the packet delivery ratio, approximately $259 \%$ compared to JARR, $58.8 \%$ and $107 \%$ against GPSI and IBR, respectively. In the dense networks, when the vehicle density is 20 vehicles $/ \mathrm{km}$, the BTDAR-R is capable of successfully delivering more packets than three other routing protocols. The packet delivery ratio is improved approximately $157 \%$ compared to JARR, $26.7 \%$ and $51.1 \%$ against GPSI and IBR, respectively. This result is because the two variant routing algorithms in BTDAR take into consideration mobility factors, such as relative speed. Vehicles that move too fast to relay packets, have a congested communication storage buffer, or have unstable connectivity between neighbours are filtered during path discovery. Accordingly, the overall packet delivery success ratio is significantly improved with the BTDAR. The GPSI performs well in low traffic density as it can maintain a cache of successful paths between source and destination vehicles. The JARR and IBR both suffer lower packet delivery ratio in sparse vehicular networks. The major reason is that these protocols lack schemes to tackle problems caused by rapid changes of network topology.

Figure 9 shows performance comparisons on packet delivery ratio versus vehicle velocity. All simulated routing protocols present a continued decrease in packet delivery ratio when vehicle speeds become faster. This result implies that when the vehicle speed is very fast, the packet delivery path becomes unstable due to the rapid change of network topology. In particular, in a sparse traffic vehicular network, packet delivery ratio is severely affected by vehicle speed. The communication between the source and destination vehicles is intermittent because of the scarce availability of relay vehicles. However, the BTDAR-R and BTDAR-P still outperforms three other counterparts routing protocols. With the BTDAR-R, when the vehicle velocity is $30 \mathrm{~m} / \mathrm{s}$, the packet delivery ratio is improved approximately $105 \%$ compared to JARR. The BTDAR is a traffic density awareness

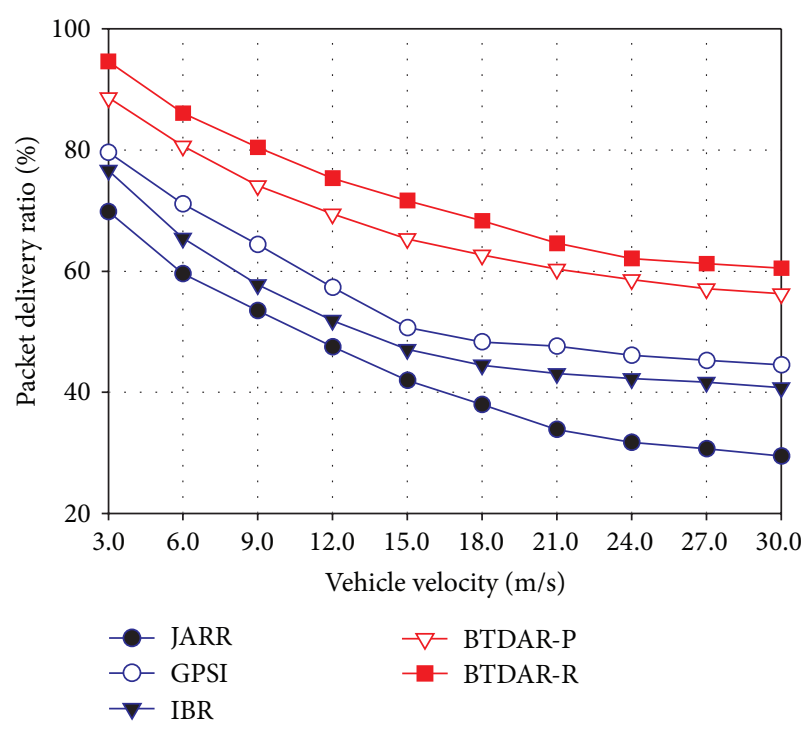

FIgURE 9: Packet delivery ratio as a function of vehicle velocity.

routing protocol that establishes a stable path between source and destination vehicles. All candidate delivery paths are compared to avoid any unstable routes and thus packet delivery ratio is significantly improved.

5.3. Number of Control Bytes Transmitted per Data Byte Delivered. Instead of the pure control overhead, we choose a ratio of control bytes transmitted and data byte delivered to explore how efficiently control packets are utilized in forwarding data in VANETs. Generally speaking, the ratio of control bytes transmitted and data byte delivered is the lower the better. Figure 10 shows the number of control bytes transmitted per data byte delivered as a function of average vehicle density. The control overheads of all routing protocols become larger as vehicle number increases. Since there is no mobility prediction in the JARR, thus the ratio for the JARR does not increase as the vehicle velocity increase. The GPSI periodically switches from the greedy mode to predictive mode and generates more control overhead adapts to vehicle number change. Our proposed BTDAR also shows more overhead in a dense traffic networks. This is due to increasing number of vehicles will increase number of control message traffic for route packets. However, the overall performance for BTDAR is still better than the other compared routing protocols.

In Figure 11, we can see that GPSI and IBR have higher ratios than JARR and BTDAR with the increase of vehicle speed. More control packets are generated and transmitted to adapt vehicle speed and build packet delivery path in GPSI and IBR, when the vehicles are moving at high speeds. The BTDAR also presents gradual increases of ratio similar to other three protocols. The BTDAR shows a better performance adapts to average vehicle speed.

5.4. Number of Total Packets Transmitted per Data Packet Delivered. We use the metric of total packets transmitted per 


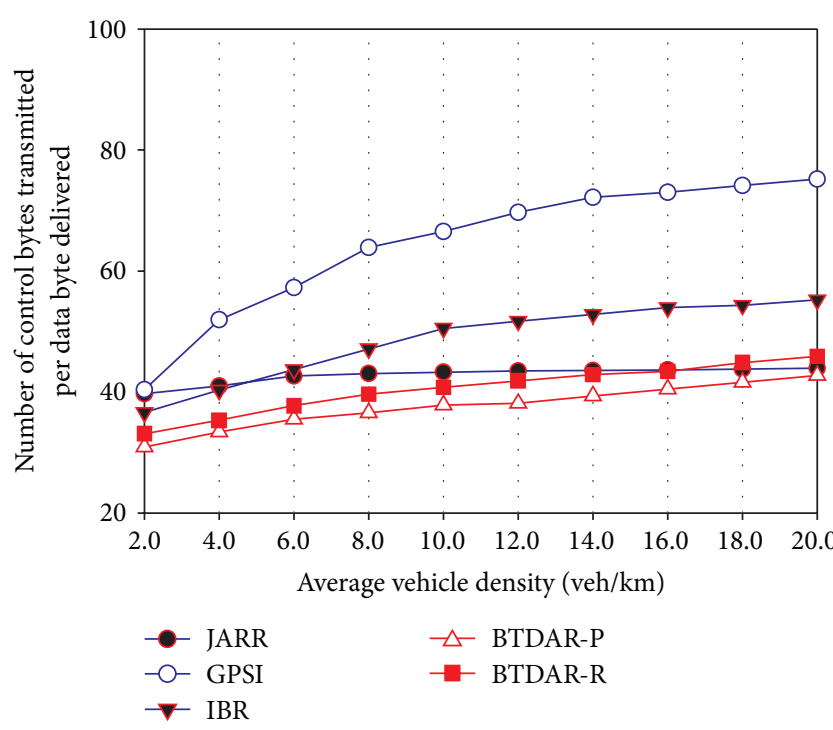

FIGURE 10: Number of control bytes transmitted per data byte delivered as a function of average vehicle density.

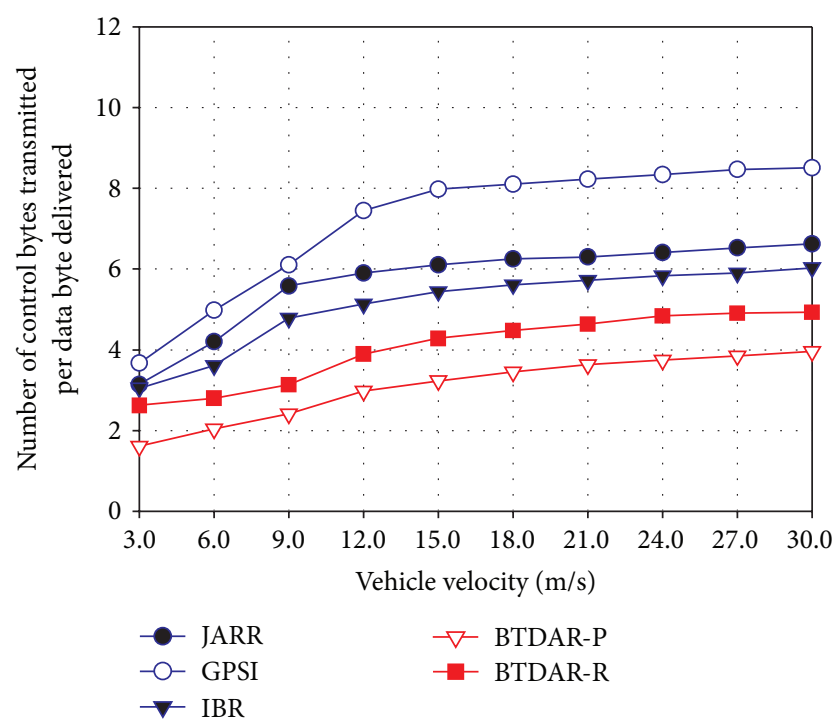

FIGURE 11: Number of control bytes transmitted per data byte delivered as a function of vehicle velocity.

data packet delivered to investigate the efficiency of routing protocol in terms of channel access in a contention-based wireless ad hoc networks. Routing protocols having the lower ratio of total packets transmitted per data packet delivered show better performance. That means control packets are not generated too much to affect the efficiency of data packets delivery when vehicle mobility pattern is changed. From Figures 12 and 13, our proposed two BTDAR protocols have lower ratio than the GPSI and IBR when the vehicle density and speed increase. The number of total packets of JARR remains almost unchanging as reasons described above. For GPSI, IBR, and BTDAR, since the amount control packet and the number of data packet delivered both increase as the

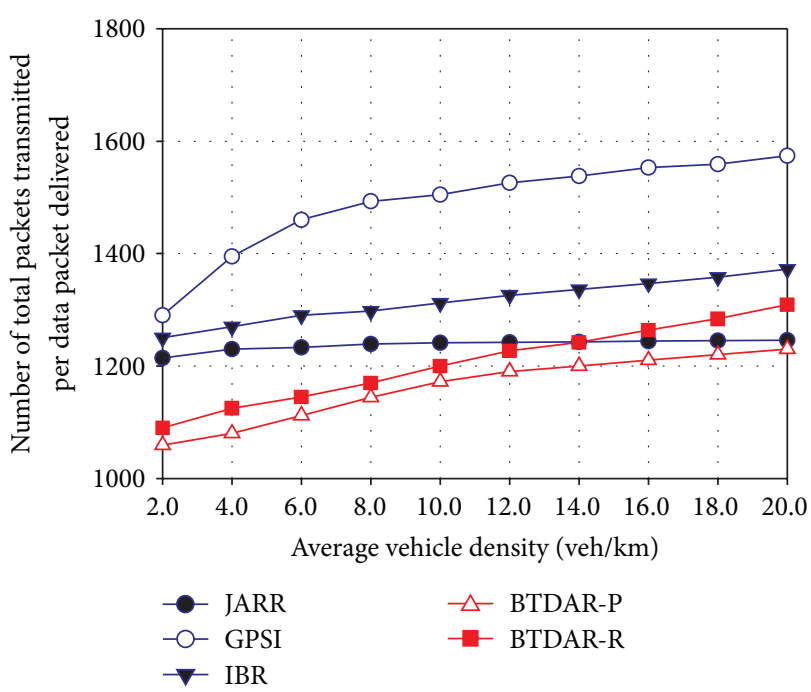

FIGURE 12: Number of total packets transmitted per data packet delivered as function of vehicle density.

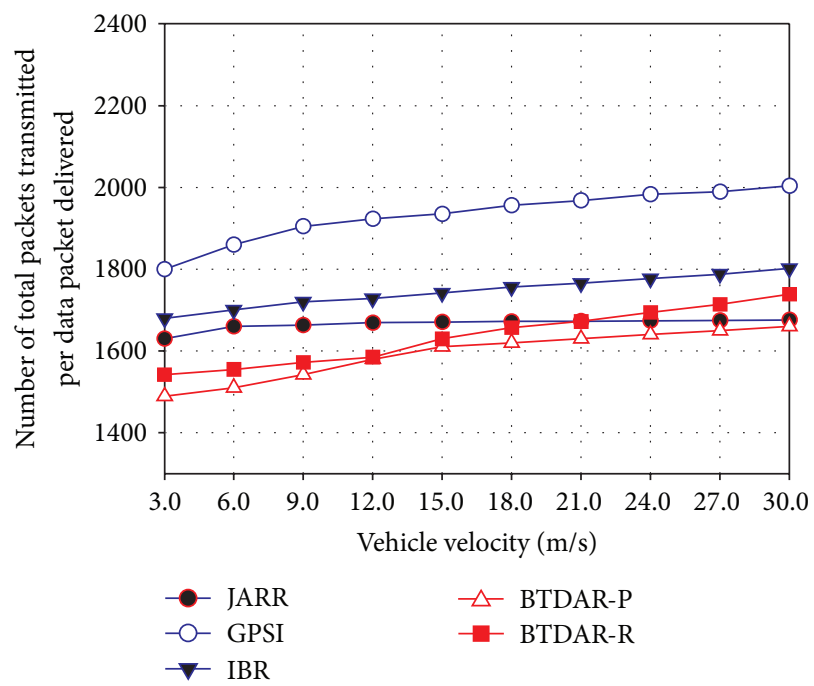

FIGURE 13: Number of total packets transmitted per data packet delivered as function of vehicle velocity.

vehicle density and speed increase, the GPSI delivers a higher portion of control message in order to adapt to vehicle density increasing. Thus the GPSI shows the higher ratio of total packets transmitted per data packet delivered. Our proposed BTDAR can deliver data along stable route than the GPSI and IBR; therefore, more data packet is transmitted to the destination. Accordingly, the overall number of BTDAR is lower than the GPSI and IBR. Thus, our proposed the BTDAR has better channel access efficiency over GPSI and IBR.

\section{Conclusions}

This paper presents an intelligent Bipolar Traffic Density Awareness Routing (BTDAR) protocol with two variants for dense and sparse traffic conditions in vehicular ad hoc 
networks. The major contribution of the proposed BTDAR is summarized as follows. (1) The intervehicle communication connectivity model is proposed with analysis of three important factors that affect packet delivery path discovery. (2) A link stability based routing protocol and min-delay based routing protocol are proposed to find an optimal packet delivery path in dense and sparse traffic vehicular networks, respectively. In the dense vehicular networks, an optimal packet delivery path is selected based on computation of path quality matrix and comparison. In the sparse networks, packet forwarding delay and carrying delay are calculated with considering effects of light traffic density to find an optimal route. (3) BTDAR is a traffic density awareness routing protocol that has intelligent characteristics. The two variants of BTDAR protocols flexibly adapt to various traffic densities of vehicular networks. Extensive simulations examined the performance of the proposed BTDAR variants. Simulation results show that the two routing algorithms outperform other three existing VANET routing protocols in terms of packet delivery delay and packet delivery ratio. Therefore, the proposed solution provides an alternative to satisfy new service requirements of routing protocols in VANETs.

\section{Abbreviation}

S: $\quad$ The average intervehicle space

$\alpha: \quad$ Effective vehicle length

$\beta$ : $\quad$ Drivers' interaction time

$\gamma$ : $\quad$ The reciprocal of vehicle maximum deceleration

$V: \quad$ Real-time vehicle velocity

$\bar{X}_{t}: \quad$ The interarrival time of vehicles that pass a certain monitor on the road

$\lambda_{1}$ : The interarrival rate of vehicles that pass a certain monitor on the road

$V_{r}: \quad$ The relative intervehicle velocity

$\theta: \quad$ Relative angle between two vehicles

$S_{d}: \quad$ The line of sight distance of two vehicles

$V_{r_{-} t h}: \quad$ Minimum threshold value of relative intervehicle velocity $V_{r}$

LLT: The link lifetime of two vehicles communication

LLT $_{\max }$ : The maximum threshold value of LLT

$W_{q}(t)$ : The cumulative distribution function of the average waiting time of a packet in the buffer queue

$W_{\max }: \quad$ The maximum predefined packet waiting time in the buffer queue

$P_{q}: \quad$ The probability that the waiting time $W_{q}$ of a packet exceeds the maximum predefined waiting time $W_{\max }$ of a specific queue

$\lambda_{2}$ : The arrival rate of the packet to the buffer queue

$\mu$ : The service rate of processing the packet out the buffer queue

c: $\quad$ The number of service channels that process the packet

$P_{q_{-} \text {min }}:$ The minimum threshold of $P_{q}$

M: $\quad$ Packet delivery path quality matrix
A: Path quality factor threshold matrix

$n_{r}$ : The total number of relay nodes on a candidate path between the source and destination vehicles

$l$ : The road length

$l_{f}$ : Packet forwarding distance

$l_{c}$ : Packet carrying distance

$P_{c}$ : The contacted probability that there is a relay vehicle connected with the source vehicle

n: The total number of packet forwarding times

$x$ : The current position of the relay vehicle at a certain time $t$

$R$ : Vehicle communication range

$A_{f}$ : Packet forwarding region

$A_{i}$ : Region of interest

$t_{1}$ : The time that the vehicle enters the area of region of interest

$t_{2}$ : The time that the vehicle leaves the area of region of interest

$d_{c}$ : Packet carrying delay between the relay vehicle and destination vehicle

$d_{q}$ : Packet forwarding delay between the relay vehicle and destination vehicle.

\section{Conflict of Interests}

The authors declare that there is no conflict of interests regarding the publication of this paper.

\section{Acknowledgments}

This work was partially supported by Ministry of Science and Technology, Taiwan R. O. C. under Grants nos. NSC 1002218-E-008-012-MY3, 102-2221-E-008-039-MY3, and 1032221-E-008-090-MY3.

\section{References}

[1] ABI Research,2013,http://www.reuters.com/article/2012/07/03/ idUS168107+03-Jul-2012+BW20120703.

[2] M. Di Felice, K. R. Chowdhury, and L. Bononi, "Cognitive radio vehicular ad hoc networks: design, implementation, and future challenges," in Mobile Ad Hoc Networking: Cutting Edge Directions, pp. 619-644, John Wiley \& Sons, Hoboken, NJ, USA, 2013.

[3] T. Delot, S. Ilarri, S. Lecomte, and N. Cenerario, "Sharing with caution: managing parking spaces in vehicular networks," Mobile Information Systems, vol. 9, no. 1, pp. 69-98, 2013.

[4] Y. Ni, Z. Yuan, and W. Liu, "Study of intelligent transportation management systems on urban energy and environment," in Informatics and Management Science V, pp. 247-255, Springer, London, UK, 2013.

[5] C.-J. Huang, I.-F. Chen, K.-W. Hu, H.-Y. Shen, Y.-J. Chen, and D.-X. Yang, "A load balancing and congestion-avoidance routing mechanism for teal-time traffic over vehicular networks," Journal of Universal Computer Science, vol. 15, pp. 2506-2527, 2009. 
[6] B. Jarupan and E. Ekici, "Location- and delay-aware cross-layer communication in V2I multihop vehicular networks," IEEE Communications Magazine, vol. 47, no. 11, pp. 112-118, 2009.

[7] I. Stojmenovic, "Simulations in wireless sensor and ad hoc networks: matching and advancing models, metrics, and solutions," IEEE Communications Magazine, vol. 46, no. 12, pp. 102$107,2008$.

[8] S. Zeadally, R. Hunt, Y.-S. Chen, A. Irwin, and A. Hassan, "Vehicular ad hoc networks (VANETS): status, results, and challenges," Telecommunication Systems, vol. 50, no. 4, pp. 217241, 2012.

[9] T. Delot, N. Mitton, S. Ilarri, and T. Hien, "GeoVanet: a routing protocol for query processing in vehicular networks," Mobile Information Systems, vol. 7, no. 4, pp. 329-359, 2011.

[10] J. Nzouonta, N. Rajgure, G. Wang, and C. Borcea, "VANET routing on city roads using real-time vehicular traffic information," IEEE Transactions on Vehicular Technology, vol. 58, no. 7, pp. 3609-3626, 2009.

[11] J. Toutouh, J. García-Nieto, and E. Alba, "Intelligent OLSR routing protocol optimization for VANETs," IEEE Transactions on Vehicular Technology, vol. 61, no. 4, pp. 1884-1894, 2012.

[12] J. Yoo, S. Choi, and C.-K. Kim, "The multi-copy diversity for routing in sparse vehicular ad hoc networks," Telecommunication Systems, vol. 50, no. 4, pp. 297-309, 2012.

[13] E. Spaho, L. Barolli, G. Mino, F. Xhafa, V. Kolici, and R. Miho, "Implementation of CAVENET and its usage for performance evaluation of AODV, OLSR and DYMO protocols in vehicular networks," Mobile Information Systems, vol. 6, no. 3, pp. 213-227, 2010.

[14] H. Mousannif, I. Khalil, and S. Olariu, "Cooperation as a service in VANET: implementation and simulation results," Mobile Information Systems, vol. 8, no. 2, pp. 153-172, 2012.

[15] W. Chen, R. K. Guha, T. J. Kwon, J. Lee, and Y.-Y. Hsu, "A survey and challenges in routing and data dissemination in vehicular ad hoc networks," Wireless Communications and Mobile Computing, vol. 11, no. 7, pp. 787-795, 2011.

[16] X. Ma, M. T. Sun, G. Zhao, and X. Liu, "An efficient path pruning algorithm for geographical routing in wireless networks," IEEE Transactions on Vehicular Technology, vol. 57, no. 4, pp. 24742488, 2008.

[17] T.-Y. Wu, Y.-B. Wang, and W.-T. Lee, "Mixing greedy and predictive approaches to improve geographic routing for VANET," Wireless Communications and Mobile Computing, vol. 12, no. 4, pp. 367-378, 2012.

[18] K. Shafiee and V. C. M. Leung, "Connectivity-aware minimumdelay geographic routing with vehicle tracking in VANETs," Ad Hoc Networks, vol. 9, no. 2, pp. 131-141, 2011.

[19] J. Bernsen and D. Manivannan, "RIVER: a reliable intervehicular routing protocol for vehicular ad hoc networks," Computer Networks, vol. 56, no. 17, pp. 3795-3807, 2012.

[20] M. H. Eiza and Q. Ni, "An evolving graph-based reliable routing scheme for VANETs," IEEE Transactions on Vehicular Technology, vol. 62, no. 4, pp. 1493-1504, 2013.

[21] J. Jeong, S. Guo, Y. Gu, T. He, and D. H. C. Du, “TSF: trajectorybased statistical forwarding for infrastructure-to-vehicle data delivery in vehicular networks," in Proceedings of the 30th IEEE International Conference on Distributed Computing Systems (ICDCS '10), pp. 557-566, IEEE Computer Society, Genova, Italy, June 2010.

[22] K. Mershad, H. Artail, and M. Gerla, "ROAMER: roadside Units as message routers in VANETs," Ad Hoc Networks, vol. 10, no. 3 , pp. 479-496, 2012.
[23] A. Fonseca and T. Vazão, "Applicability of position-based routing for VANET in highways and urban environment," Journal of Network and Computer Applications, vol. 36, no. 3, pp. 961-973, 2013.

[24] H. Saleet, R. Langar, K. Naik, R. Boutaba, A. Nayak, and N. Goel, "Intersection-based geographical routing protocol for VANETs: a proposal and analysis," IEEE Transactions on Vehicular Technology, vol. 60, no. 9, pp. 4560-4574, 2011.

[25] S. Sukumaran, L. Ramachandran, and S. R. Sunny, "Intersection based traffic aware routing in VANET," in Proceedings of the International Conference on Advances in Computing, Communications and Informatics (ICACCI '13), pp. 1414-1417, Mysore, India, August 2013.

[26] L.-D. Chou, J.-Y. Yang, Y.-C. Hsieh, D.-C. Chang, and C.-F. Tung, "Intersection-based routing protocol for VANETs," Wireless Personal Communications, vol. 60, no. 1, pp. 105-124, 2011.

[27] C. Tee and A. Lee, "Adaptive reactive routing for VANET in city environments," in Proceedings of the 10th International Symposium on Pervasive Systems, Algorithms, and Networks (ISPAN '09), pp. 610-614, IEEE, Kaohsiung, Taiwan, December 2009.

[28] H. F. Sun, G. C. Luo, and H. Chen, "JTAR: junction-based traffic aware routing in sparse urban VANETs," IEICE Transactions on Communications, vol. E95.B, no. 3, pp. 1007-1010, 2012.

[29] N. Wisitpongphan, F. Bai, P. Mudalige, V. Sadekar, and O. Tonguz, "Routing in sparse vehicular ad hoc wireless networks," IEEE Journal on Selected Areas in Communications, vol. 25, no. 8, pp. 1538-1556, 2007.

[30] Federal Highway Administration Research and Technology, 2013, http://www.fhwa.dot.gov/research/.

[31] D. Gross, J. F. Shortle, J. M. Thompson, and C. M. Harris, Fundamentals of Queueing Theory, Wiley Series in Probability and Statistics, John Wiley \& Sons, Hoboken, NJ, USA, 4th edition, 2008.

[32] G. Rill, Road Vehicle Dynamics: Fundamentals and Modeling, CRC Press, New York, NY, USA, 2012.

[33] T. Taleb, E. Sakhaee, A. Jamalipour, K. Hashimoto, N. Kato, and Y. Nemoto, "A stable routing protocol to support ITS services in VANET networks," IEEE Transactions on Vehicular Technology, vol. 56, no. 6, pp. 3337-3347, 2007.

[34] L. Zhao, F. Li, and Y. Wang, "Hybrid position-based and DTN forwarding in vehicular ad hoc networks," in Proceedings of the IEEE Vehicular Technology Conference, pp. 1-5, IEEE Computer Society Press, Quebec City, Canada, 2012.

[35] P.-C. Cheng, K. C. Lee, M. Gerla, and J. Härri, “GeoDTN+Nav: geographic DTN routing with navigator prediction for urban vehicular environments," Mobile Networks and Applications, vol. 15, no. 1, pp. 61-82, 2010.

[36] The Network Simulator-ns2, 2013, http://www.isi.edu/nsnam/ ns/.

[37] M. Behrisch, L. Bieker, J. Erdmann, and D. Krajzewicz, "SUMO-simulation of urban mobility: an overview," in Proceedings of the $3 r d$ International Conference on Advances in System Simulation (SIMUL '11), pp. 55-60, IARIA, Barcelona, Spain, October 2011.

[38] OpenStreetMap, 2013, http://www.openstreetmap.org/. 

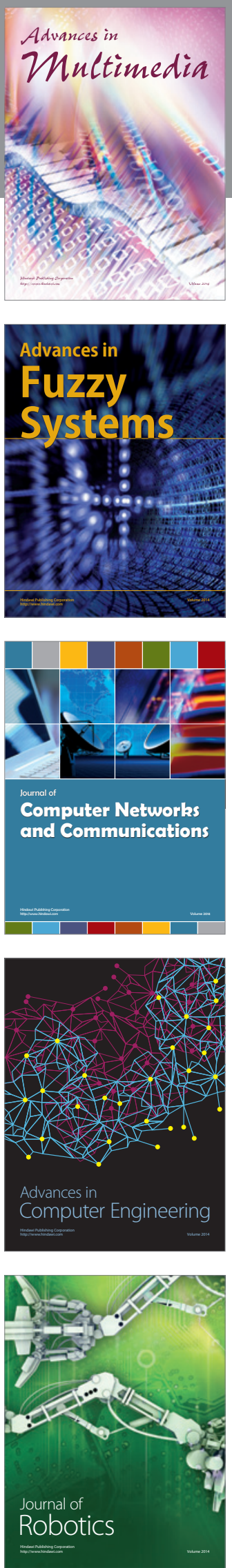

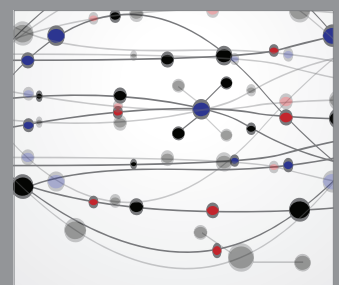

The Scientific World Journal
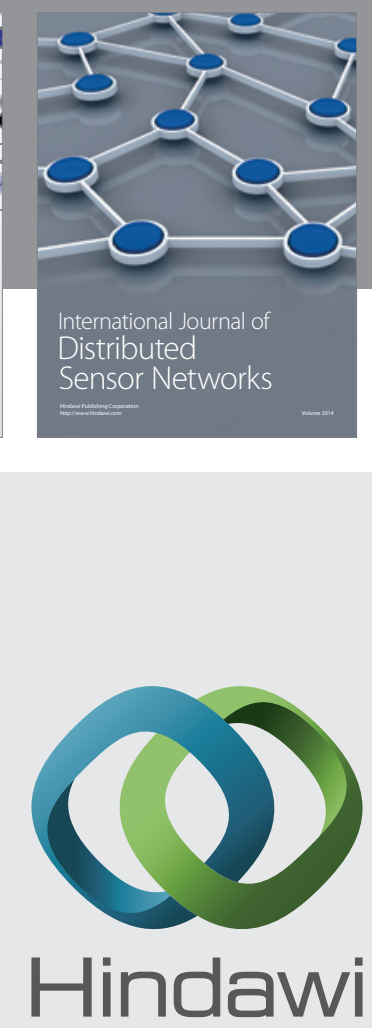

Submit your manuscripts at

http://www.hindawi.com
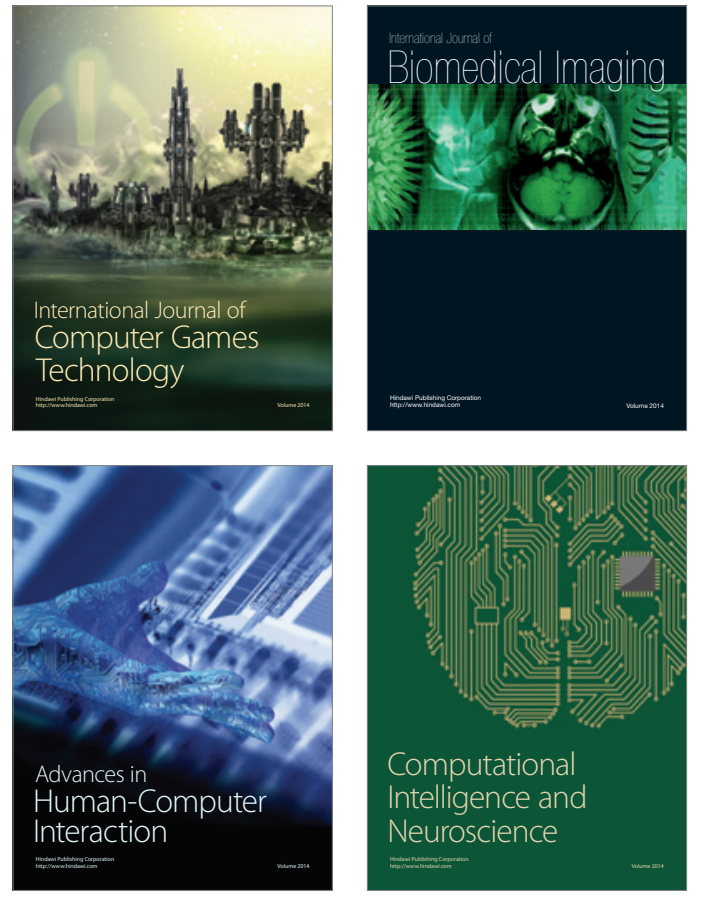
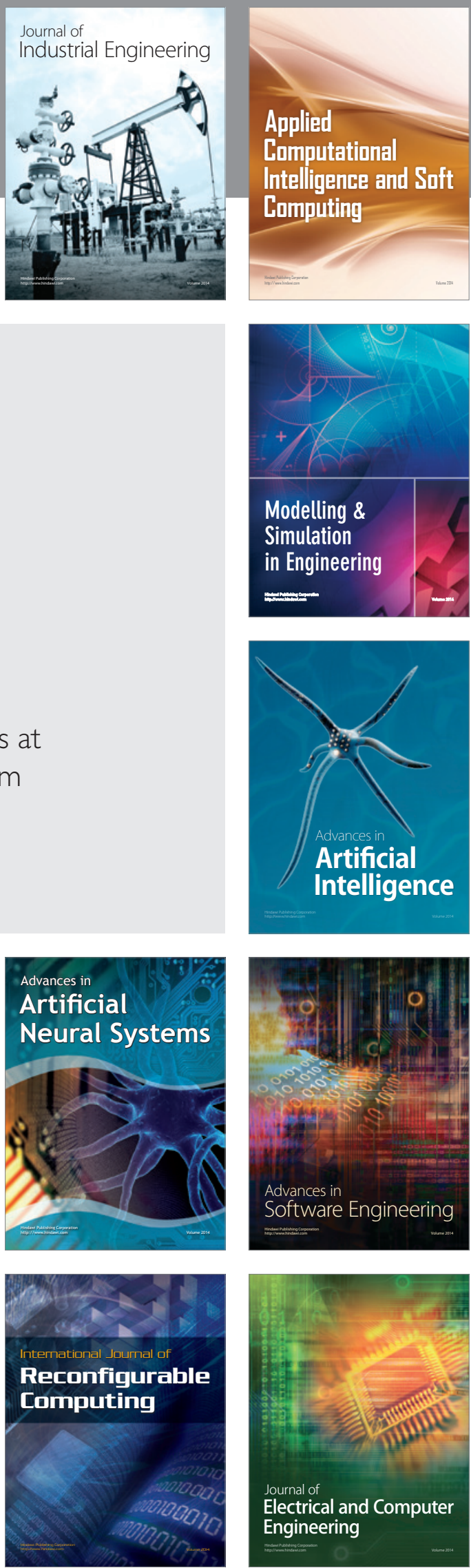